each electron all pointed in the same direction or were aligned. The question, Awschalom said, was whether a cloud or bundle of electrons all spinning the same way would retain that same spinning when the cloud is moved to an adjacent semiconducting material. When transferring an electron spin across an interface between the semiconductors $\mathrm{GaAs}$ and $\mathrm{ZnSe}$ in a magnetic field, the researchers found that the spins stayed aligned, even as the temperature of the materials was raised, in some cases, to room temperature.

Furthermore, the researchers observed that the GaAs semiconductor serves as a spin reservoir. Awschalom said that if spin was pulled from one material (e.g., $\mathrm{GaAs}$ ) to another (e.g., ZnSe), the spins in the adjacent layer acquire the original spin frequency and lifetime of the reservoir. Therefore the total transferred spin current can have the properties of either the reservoir or the adjacent layer, and an external electric field gates the transition between the two very different regimes.

Under electrical bias, the relative increase in spin-coherent injection was up to $500 \%$ in the $n$-GaAs $/ n-Z n S e$ junction. Significantly, this increase was nearly $4000 \%$ in the $p$-GaAs $/ n$-ZnSe junction. The results in the $n-n$ junction are due to the GaAs spin reservoirs whereas in the $p-n$ junction, the data suggest that there is enhancement in spontaneous transfer mechanisms. These results, particularly for the $p$ - $n$ heterostructures, could point the way toward spin transistors.

\section{Cobalt-Doped Anatase Titanium Dioxide Thin Films Behave as Room-Temperature Magnetic Semiconductors}

Scientists at Pacific Northwest National Laboratory (PNNL) have created a thinfilm semiconductor material made of titanium, oxygen, and cobalt. Their material demonstrated improvement of magnetic strength by nearly a factor of five over that currently demonstrated.

In order to be practical, spintronics will need to use semiconductors that maintain their magnetic properties at room temperature. This is a challenge because most magnetic semiconductors lose their magnetic properties above critical temperatures that are well below room temperature, and would require expensive and impractical refrigeration in order to work in an actual computer.

Scott Chambers, a chemist and PNNL senior chief scientist, and his team of scientists achieved these properties in a crystalline oxide film known as anatase titanium dioxide that is infused with a small amount of cobalt, a magnetic impurity. As described in a poster presentation at the 2001 Spintronics Workshop in Washington, D.C., in August, Chambers and his team created this magnetic semiconductor material using molecular-beam epitaxy. A team of scientists at IBM, led by research staff scientist Robin Farrow, then characterized the material's magnetic properties.

\section{Ion-Beam Mixing Used to Synthesize Cu-Ag Nanocomposites}

Using analytic modeling, atomistic simulations, and experiments, researchers from the University of Illinois at UrbanaChampaign (UIUC) have proposed the use of ion-beam mixing with controlled irradiation conditions to synthesize nanocomposites. Researchers Raúl A. Enrique and Pascal Bellon determined that the nanocomposites are directly stabilized during irradiation because of a dynamical self-organization reaction.

Last year (Physical Review Letters 84 [2000] p. 2885), the researchers identified analytically that certain irradiation conditions can lead to a dynamical stabilization of nanocomposites. They later confirmed the analytical predictions by using atomistic kinetic Monte Carlo simulations (Physical Review B 63 [2001] p. 134111). In a publication this past summer (Applied Physics Letters 78 [2001] p. 4178), Enrique and Bellon demonstrated that this approach works by synthesizing $\mathrm{Cu}-\mathrm{Ag}$ nanocomposites using 1-MeV Kr irradiations.

"In fact," said Bellon, "the two phases are mixed at such a fine scale that the decomposition cannot be directly seen by electron microscopy imaging techniques."

According to the researchers, the selforganization reaction results because the various dynamical processes operating during irradiation occur at different length scales. The researchers predict that the length scale of these nanocomposites can be continuously tuned by varying the irradiation conditions, such as the irradiation temperature.

"This would be a very important point for the synthesis of optimized nanocomposites, which almost always require a tight control of grain or phase size," said Bellon.

In a separate study, researchers G.C. Rizza and H. Bernas from CNRS in France and M. Strobel and K.-H. Heinig from Forschungzentrum Rossendorf in Germany reported related results on the stabilization of nanoprecipitates in irradiated $\mathrm{SiO}_{2}$ with gold inclusions (Nuclear Instruments and Methods in Physics Research B 178 [2001] p. 78). They are currently applying this method to synthesize active dots in thin films for optical and
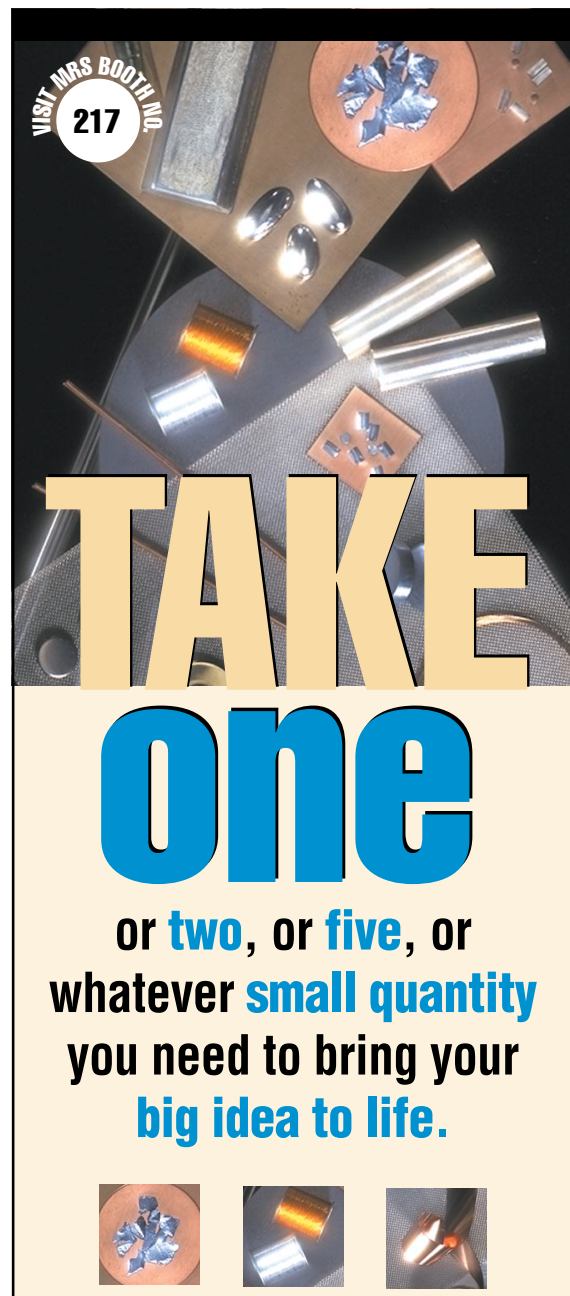

Choose from more than

40,000 different items, in stock and ready for immediate shipment.

\section{metals - alloys - polymers ceramics " composites}

We understand that little things mean a lot when you're on to something big. Contact us today to find out what we can offer you.

Web: www.goodfellow.com Real live person: 1-800-821-2870 E-mail: info@goodfellow.com Fax: 1-800-283-2020

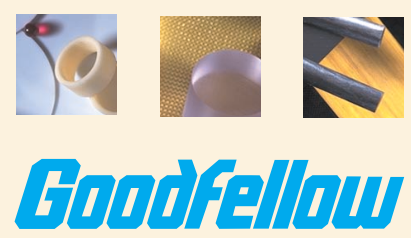

800 Lancaster Ave., Berwyn, PA 19312-1780

Circle No. 14 on Inside Back Cover 